\title{
Risk of decompression illness among 230 divers in relation to the presence and size of patent foramen ovale
}

\author{
Sandra Rea Torti, Michael Billinger, Markus Schwerzmann, Rolf Vogel, \\ Rainer Zbinden, Stephan Windecker, Christian Seiler*
}

Department of Cardiology, University Hospital, CH-3010 Bern, Switzerland

Received 29 November 2003; revised 9 April 2004; accepted 13 April 2004

\section{KEYWORDS \\ Patent foramen ovale; Diving;}

Decompression illness

\begin{abstract}
Background The risk of developing decompression illness $(\mathrm{DCl})$ in divers with a patent foramen ovale (PFO) has not been directly determined so far; neither has it been assessed in relation to the PFO's size.

Methods In 230 scuba divers (age $39 \pm 8$ years), contrast trans-oesophageal echocardiography (TEE) was performed for the detection and size grading $(0-3)$ of PFO. Prior to TEE, the study individuals answered a detailed questionnaire about their health status and about their diving habits and accidents. For inclusion into the study, $\geqslant 200$ dives and strict adherence to decompression tables were required.

Results Sixty-three divers (27\%) had a PFO. Overall, the absolute risk of suffering a $\mathrm{DCl}$ event was 2.5 per $10^{4}$ dives. There were 18 divers (29\%) with, and 10 divers $(6 \%)$ without, PFO who had experienced $\geqslant 1$ major $\mathrm{DCl}$ events $(P=0.016)$. In the group with PFO, the incidence per $10^{4}$ dives of a major $\mathrm{DCl}$, a DCl lasting longer than $24 \mathrm{~h}$ and of being treated in a decompression chamber amounted to 5.1 (median 0 , interquartile range [IQR] 0-10.0), 1.9 (median 0, IQR 0-4.0) and 3.6 (median 0, IQR $0-9.8)$, respectively and was $4.8-12.9$-fold higher than in the group without PFO $(P<0.001)$. The risk of suffering a major $\mathrm{DCl}$, of a DCl lasting longer than $24 \mathrm{~h}$ and of being treated by recompression increased with rising PFO size.

Conclusion The presence of a PFO is related to a low absolute risk of suffering five major $\mathrm{DCl}$ events per $10^{4}$ dives, the odds of which is five times as high as in divers without PFO. The risk of suffering a major DCI parallels PFO size.

(C) 2004 The European Society of Cardiology. Published by Elsevier Ltd. All rights reserved.
\end{abstract}

\section{Introduction}

Most of the medical health problems in scuba (self-contained underwater breathing apparatus) diving are consequences of decompression during the ascent of the diver. The term decompression illness $(\mathrm{DCl})$ is used for describing decompression disorders with gas bubbles as the initiator (i.e., decompression sickness developing

\footnotetext{
* Corresponding author. Tel.: +41-31-632-36-93; fax: +41-31-632-42 99.

E-mail address: christian.seiler.cardio@insel.ch (C. Seiler).
}

locally from expanding gas nuclei or arterial gas embolism). Such gas bubbles are thought to develop by expansion of pre-existing gas nuclei found at normal atmospheric pressure in joints, the spine, sweat glands and skin pores. ${ }^{1,2}$ These tissue bubbles may gain access to the capillary or lymphatic bed by migration, and thus, can enter the venous circulatory system. Small gas volumes are filtered by the lungs and exhaled, large ones may cause pulmonary barotrauma and/or may escape into the systemic circulation via pulmonary arterio-venous shunts. An alternative pathway for venous gas bubbles to be transferred to the systemic arterial side 
(i.e., gas embolism by definition) consists of an intraatrial shunt due to an atrial septal defect or a patent foramen ovale (PFO). Of all possibilities for arterialisation of venous gas bubbles, a PFO is likely to be the most prevalent passage, because PFOs are found in $1 / 4$ to $1 / 3$ of the general population, ${ }^{3}$ whereas atrial septal defects and pulmonary vascular malformations are much rarer.

It has been indicated already in 1986 that a cardiac right-to-left shunt may be important for paradoxical gas embolism in scuba divers. ${ }^{4}$ Subsequently, the importance of PFO for decompression events in divers has been further investigated. ${ }^{5-7}$ However, the risk of developing $\mathrm{DCl}$ when a diver has a PFO has not been directly and accurately determined so far, but only crudely estimated on the basis of a meta-analysis ${ }^{8}$ incorporating three partly small studies which, in addition, have employed transthoracic instead of the more sensitive trans-oesophageal echocardiography (TEE) for detecting PFO. ${ }^{6,9,10}$

Therefore, the purpose of this investigation in 230 mostly sport divers was to assess the absolute and relative odds of $\mathrm{DCl}$ events with or without subsequent treatment in a decompression chamber in relation to the presence and size of a PFO as characterised by TEE.

\section{Methods}

\section{Study subjects}

Contrast TEE was performed in 230 scuba divers (age $39 \pm 8$ years). Prior to TEE, the study individuals answered a detailed questionnaire about their health status and about their diving habits and accidents. The physician performing the TEE was unaware of the questionnaire's content or of the diving history. For inclusion into the study, $\geqslant 200$ dives and adherence to decompression tables were required. Thus, the present work concerns unexpected $\mathrm{DCl}$. Subsequently, the more general term $\mathrm{DCl}$ is used instead of decompression sickness and arterial gas embolism (the two subunits of $\mathrm{DCl}$ ) which, purely based on self reported temporal information on the $\mathrm{DCl}$, are clinically rather difficult to distinguish. Data from 52 of the 230 divers have been published, in part, elsewhere. ${ }^{11}$ The study population was divided in two groups according to the presence of a PFO as detected by contrast TEE.

The study was approved by the local ethics committee, and the study subjects gave written informed consent to participate in the study.

\section{Health status, diving habits and accidents}

Before examining the study subjects by TEE, their past medical history, including medication, alcohol use and smoking habits were assessed. Special attention was given to a history of cardiac disease, systemic hypertension, diabetes mellitus, asthma, migraine or chronic headache, other neurologic disorders, rheumatic illness, previous operations and/or non-diving related accidents. From each diver a detailed diving history was obtained, including total number of dives, duration of engagement in diving, mean diving depth, number of dives $\geqslant 40 \mathrm{~m}$, breathing gas used (compressed air or higher-thannormal partial pressure of oxygen plus nitrogen or helium) and method of pressure equilibration in the middle ear. The presence or absence of $\mathrm{DCl}$ and, if having been present, signs and symptoms of $\mathrm{DCl}$ were enquired. $\mathrm{A} \mathrm{DCl}$ event was classified as a minor or major $\mathrm{DCl}$ event. Minor $\mathrm{DCl}$ events were scored (0-3) according to the frequency of their occurrence (never, rarely, every fourth to third dive, $\geqslant$ every second dive). Minor DCl symptoms included bends, cutaneous erythema, extreme fatigue, headache, dizziness, paraesthesias and tinnitus. Major $\mathrm{DCl}$ events were defined by one or more of the following symptoms: limb weakness, cutaneous sensory level, impaired bowel or bladder control, paresis or paraplegia, blurred vision, dysarthria, amnesia for the event, hemiplegia or loss of consciousness after a dive. The time frame of occurrence and disappearance of $\mathrm{DCl}$ symptoms and eventual treatment in a decompression chamber was also registered. At the time of answering the questionnaire all divers were unaware of whether they had a PFO or not.

\section{Contrast trans-oesophageal echocardiography}

Before intubation of the TEE probe, the epipharynx was anaesthetised using lidocaine hydrochloride 10\% spray. A 3-lead ECG and blood pressure were registered during TEE. No sedative medication was employed in any of the study subjects. TEE was performed in the left lateral supine position of the study subject using a Siemens Acuson Sequoia ${ }^{\circledR}$ C256 (Mountain View, CA, USA) Doppler echocardiography system with a multi-plane, 3.5-7 MHz probe. Examination for the presence or absence of PFO occurred in the transversal $\left(0-30^{\circ}\right)$ and longitudinal $\left(90^{\circ}\right.$; Fig. 1) image plane. The echo-contrast medium for the detection of a right-to-left atrial shunt consisted of an ad hoc sonicated mixture of $0.2 \mathrm{ml}$ of air and $1.8 \mathrm{ml}$ of a gelatine containing plasma expander (Physioge ${ }^{\circledR}$ ). Echo-contrast tests were performed in the two image planes mentioned above by injection of the $2 \mathrm{ml}$ of contrast into the right antecubital vein. Using the Valsalva manoeuvre (strain phase starting simultaneously with the contrast bolus injection), a left-ward deviation of the interatrial septum in the fossa ovalis region (Fig. 1(a) and (b)) was observed immediately after release of the Valsalva strain phase (lasting 5-10 s); this was observed in all individuals and was taken as a sign of a successful Valsalva manoeuvre (short right atrial pre-load increase and pressure rise). The diagnosis of PFO required the crossing of bubbles from the right to the left atrium (Fig. 1(c) and (d)) within four heart beats following release of the Valsalva strain phase. Otherwise, appearance of bubbles in the left atrium was considered transpulmonary. ${ }^{12}$ The degree of PFO was qualitatively characterised by a score of $0-3$, with a score of 1 representing the crossover of a few single bubbles, and a score of 3 representing the shunt of an entire cloud of bubbles (Fig. 1(c) and (d); score 2 between 1 and 3 ).

\section{Statistical analysis}

Incidence rates of $\mathrm{DCl}$ events were calculated by analysing individual DCI rates. An unpaired two-tailed Student's $t$-test was used for comparison of normally distributed variables between two groups. Inter-group comparison of non-normal data was performed by a two-tailed Mann-Whitney $U$ rank-sum test. Intergroup comparison of categorical data was done by a two-tailed Fisher's exact test. The risk inherent to the presence of a PFO and its corresponding $95 \%$ confidence interval was analysed with a logistic regression model for dichotomous outcomes ( $\mathrm{DCl}$ event lasting more than $24 \mathrm{~h}$ or stay in decompression chamber) and with Poisson regression for count data ( $\mathrm{DCl}$ events). The presence of a PFO, the number of dives, the breathing gas, pressure equilibration and the number of dives deeper than $40 \mathrm{~m}$ were tested as predictors of $\mathrm{DCl}$ events in a multivariate Poisson regression model. Stepwise backwards elimination of non-significant 

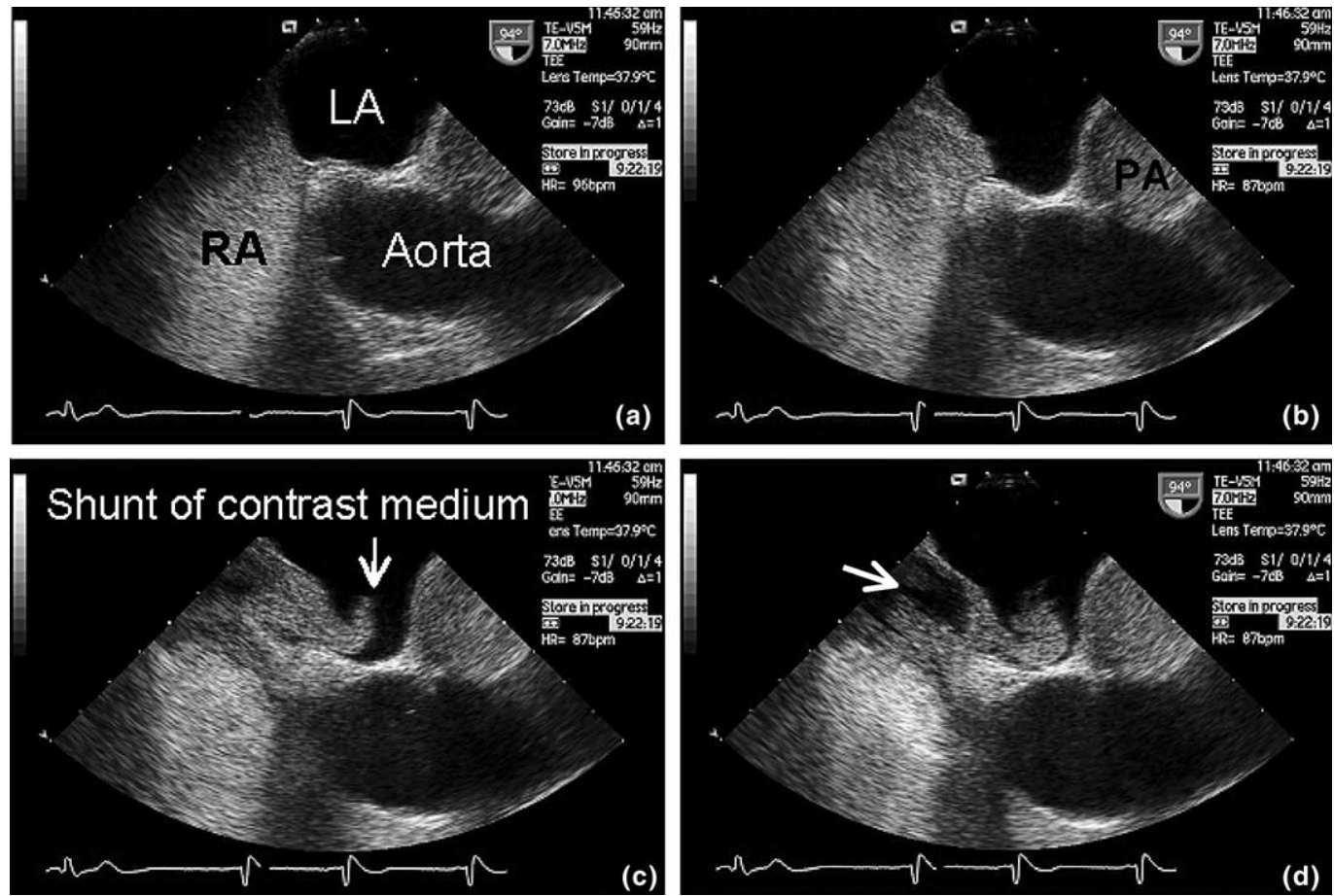

Fig. 1 Trans-oesophageal contrast echocardiography (TEE) for the detection of patent PFO. Panel a: TEE long axis view (right side: cranial; left side: caudal) showing the left atrium (LA) and the aortic root free of ultrasound contrast medium as well as the right atrium (RA) filled with contrast bubbles. The image is taken close to the end of the Valsalva strain phase with the inter-atrial septum bulged towards the RA. Panel b: Identical TEE image plane as in all other panels (long axis view) taken immediately after release of the Valsalva strain phase. The inter-atrial septum (fossa ovalis region) now bulges towards the LA, thus indicating a pressure rise in the RA above that in the LA. PA: pulmonary artery. Panel c: Long axis view image obtained instantaneously after the image shown in panel b revealing a shunt of contrast medium across a PFO from the right to the left atrium. Panel d: The shunt is even more pronounced on this next image (PFO grade 3). Washout of contrast medium in the RA is visible (arrow), which is caused by the inflow of contrastfree blood from the inferior vena cava.

predictors was performed at $P>0.1$. Analysing the variance of the residuals of the number of predicted versus observed DCls with this model, no evidence for overdispersion was found. Subsequently, we separately recalculated the risk of any $\mathrm{DCl}$ event (major $\mathrm{DCl}, \mathrm{DCl}$ lasting more than $24 \mathrm{~h}$, stay in decompression chamber) adjusted to the number of dives. For calculation of the risk ratio of decompression events depending on PFO size (Table 3), the categorical variable PFO size was extended into a dummy-variable set. Data are expressed as mean value \pm standard deviation for normally distributed data or as median \pm inter-quartile range for non-normal data. To allow comparison with previous reports incidence rates are given as mean values, even if they were not normally distributed. Statistical significance was defined at a $P$-value $<0.05$. Data were analysed using STATA 5.0 statistical software (Stata Corp., TX, USA).

\section{Results}

\section{Study subjects, health status and diving habits}

Of all 230 study individuals, 63 (27\%) had a PFO as detected for the first time by TEE and 167 (73\%) had no PFO (Table 1). Divers with and without PFO did not differ with regard to age, gender, weight, height and systemic blood pressure (Table 1).

There were no difference between the two study groups regarding alcohol use, smoking, heart disease, respiratory disease, diabetes mellitus, systemic hypertension, other health problems, or daily medication (Table 1). The frequency of migraine or chronic headache was higher in divers with, rather than in those without, PFO.

The total number of dives among subjects with PFO was higher than in those without PFO (Table 2). The diving experience in years was similar in individuals both with and without PFO, as well the average diving depth. The number of deep dives to depths $\geqslant 40 \mathrm{~m}$ below water surface was higher in subjects with, rather than in those without, PFO. As a consequence, divers with PFO used compressed air as breathing gas less often (i.e., more often with so-called nitrox gas with a higher-than-normal partial pressure of oxygen (Table 2).

\section{Diving accidents and their treatment in relation to PFO}

Overall, the absolute risk of suffering any $\mathrm{DCl}$ was 2.5 per $10^{4}$ dives. The minor $\mathrm{DCl}$ score was significantly higher in the group with, rather than without, PFO (Table 2). There were 18 divers (29\%) with and 10 divers (6\%) without PFO who had previously experienced 1 or more major $\mathrm{DCl}$ events $(P<0.001$; Table 2$)$; the divers with $>1$ serious $\mathrm{DCl}$ were exclusively in the PFO group with 1 diver having 2 and 1 diver experiencing three 
Table 1 Characteristics of study subjects

\begin{tabular}{llll}
\hline & PFO & $\varnothing$ PFO & $P$ \\
\hline Number of divers & 63 & 167 & \\
Age (years) & $40 \pm 8$ & $38 \pm 8$ & 0.30 \\
Men (\%) & $53(84)$ & $131(78)$ & 0.34 \\
Alcohol use daily (\%) & $6(10)$ & $15(9)$ & 0.90 \\
Weight (kg) & $71 \pm 12$ & $72 \pm 14$ & 0.82 \\
Height (cm) & $175 \pm 8$ & $173 \pm 11$ & 0.81 \\
Systemic blood pressure (mm Hg) & $120 / 71$ & $118 / 69$ & 0.61 \\
Smoking (\%) & $20(32)$ & $44(26)$ & 0.42 \\
Diabetes mellitus (\%) & $1(2)$ & $3(2)$ & 0.91 \\
Systemic hypertension (\%) & $3(5)$ & $10(6)$ & 0.72 \\
Heart disease (\%) & $3(5)$ & $3(2)$ & 0.35 \\
Respiratory disease (\%) & $2(3)$ & $4(3)$ & 0.67 \\
Joint symptoms (\%) & $6(10)$ & $13(7)$ & 0.79 \\
Medication use daily (\%) & $6(10)$ & $21(13)$ & 0.65 \\
Migraine or chronic headache (\%) & $11(17)$ & $10(6)$ & 0.011 \\
\hline
\end{tabular}

Abbreviations: PFO: patent foramen ovale; $\varnothing \mathrm{PFO}$ : no patent foramen ovale.

events. The symptoms of all major $\mathrm{DCl}$ events began shortly before, or within 30 min after, surfacing. In the PFO group, 12 (19\%) divers had to be treated in the decompression chamber, whereas the corresponding number was $3(2 \%)$ in the group without PFO $(P<0.001)$. None of the divers were in a decompression chamber more than once or had more than one $\mathrm{DCl}$ lasting for longer than $24 \mathrm{~h}$.

In the group with PFO, the incidence per $10^{4}$ dives of suffering a major $\mathrm{DCl}$, a major $\mathrm{DCl}$ lasting longer than 24 $\mathrm{h}$ and of being treated in the decompression chamber amounted to 5.1 (median 0, IQR 0-10.0), 1.9 (median 0, IQR 0-4.0) and 3.6 (median 0, IQR 0-9.8), respectively, and was significantly higher than in the group without PFO (Table 2, Fig. 2). The respective relative risk for individuals with, versus those without, PFO was equal to a factor of $4.8(95 \% \mathrm{Cl} 2.3-10.1), 5.7(95 \% \mathrm{Cl} 2.0-16.1)$,

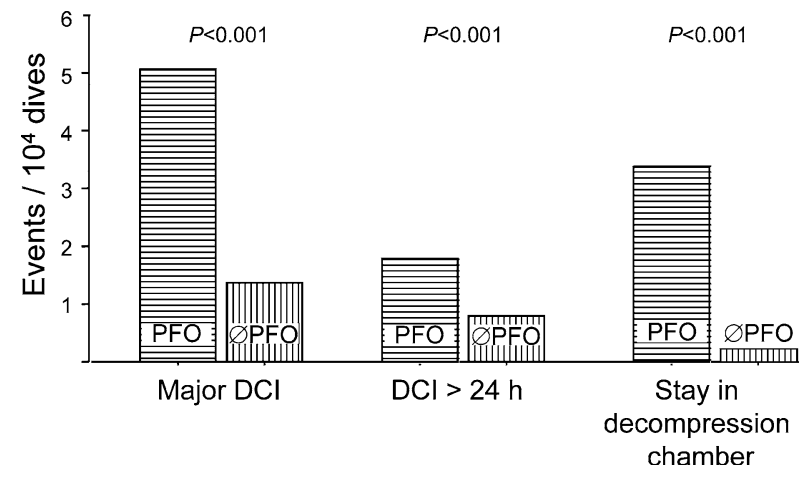

Fig. 2 Mean number of $\mathrm{DCl}$ events, $\mathrm{DCl}$ events lasting longer than $24 \mathrm{~h}$ and stay in the decompression chamber per $10^{4}$ dives (vertical axis) among divers with (PFO) and without patent foramen ovale ( $\varnothing \mathrm{PFO})$.

and 12.9 (95\% Cl 3.5-47.4), respectively. Using multivariate Poisson regression analysis, aside from the presence of a PFO, the number of dives was the only predictor of major $\mathrm{DCl}$ events. Correction for diving experience (i.e., number of dives) did not influence the risk associated with a PFO. Corrected PFO risk ratios were 4.5 (95\% Cl 2.1-9.3) for any major DCl event, $5.3(95 \% \mathrm{Cl}$ 1.8-15.2) for having a DCl lasting more than $24 \mathrm{~h}$ and $12.7(95 \% \mathrm{Cl} 3.3-49.3)$ for being treated in a decompression chamber.

The risk of having a major $\mathrm{DCl}$ increased with every degree of PFO (Table 3 and Fig. 3). Divers with a PFO grade 1 had a similar risk as divers without PFO $(1.1,95 \%$ $\mathrm{Cl} 0.14-8.4$ ), but divers with PFO grade 2 and grade 3 had a 4.4 -fold $(95 \% \mathrm{Cl} 1.8-10.7)$ and 6.6 -fold $(95 \% \mathrm{Cl}$ 2.8-15.5) risk of major $\mathrm{DCl}$ events compared with divers without PFO, respectively. In summary, the risk for any serious decompression event was similar in divers with no PFO or PFO grade 1, and substantially lower than in divers with PFO grade 2 or 3 (Table 3 ).

Table 2 Diving habits, decompression symptoms and patent foramen ovale

\begin{tabular}{|c|c|c|c|c|c|c|}
\hline & PFO $(n=63)$ & $\varnothing$ PFO $(n=167)$ & $P$ & Odds ratio & $95 \% \mathrm{Cl}$ & \\
\hline Number of dives per person (median, IQR) & $650(250-1200)$ & $400(214-800)$ & 0.009 & & & \\
\hline Years of diving & $11 \pm 8$ & $9 \pm 7$ & 0.25 & & & \\
\hline Diving depth (m) & $29 \pm 9$ & $28 \pm 9$ & 0.83 & & & \\
\hline Number of dives $\geqslant 40 \mathrm{~m}$ (median, IQR) & $50(10-150)$ & $40(8-100)$ & 0.10 & & & \\
\hline Compressed air as breathing gas (\%) & $41(65)$ & $136(81)$ & 0.01 & & & \\
\hline Valsalva for pressure equilibration (\%) & $20(32)$ & $39(23)$ & 0.06 & & & \\
\hline \multicolumn{7}{|l|}{ Decompression illness events $(D C I)$} \\
\hline Minor DCl score $(0-27)$ & $3.5 \pm 2.3$ & $2.6 \pm 1.9$ & $<0.01$ & & & \\
\hline \multirow{2}{*}{$\begin{array}{l}\text { Number of divers with major } \mathrm{DCl} \text { events } \\
\text { Individual event rate/ } 10^{4} \text { dives (median, IQR) }\end{array}$} & $18(29 \%)$ & $10(10 \%)$ & $<0.001$ & & & \\
\hline & $5.1(0,0-10.0)$ & $1.5(0,0-0)$ & $<0.001$ & 4.8 & 2.3 & $10.1^{\mathrm{b}}$ \\
\hline \multirow{2}{*}{$\begin{array}{l}\text { Number of divers with } \mathrm{DCl}>24 \mathrm{~h} \\
\quad \text { Individual event rate } / 10^{4} \text { dives }^{\mathrm{a}} \text { (median, IQR) }\end{array}$} & $11(17 \%)$ & $6(4 \%)$ & 0.001 & & & \\
\hline & $1.9(0,0-4.0)$ & $0.9(0,0-0)$ & $<0.001$ & 5.7 & 2.0 & $16.1^{\mathrm{c}}$ \\
\hline \multirow{2}{*}{$\begin{array}{l}\text { Number of divers with stay in } \mathrm{DC} \\
\text { Individual event rate } / 10^{4} \text { dives (median, IQR) }\end{array}$} & 12 (19\%) & $3(2 \%)$ & $<0.001$ & & & \\
\hline & $3.6(0,0-9.8)$ & $0.3(0,0-0)$ & $<0.001$ & 12.9 & 3.5 & $47.4^{c}$ \\
\hline
\end{tabular}

Abbreviations: DC: decompression chamber, IQR: Inter-quartile range, PFO: patent foramen ovale; $\varnothing \mathrm{PFO}$ : no patent foramen ovale.

${ }^{a} \mathrm{DCl}>24 \mathrm{~h}=$ decompression illness lasting longer than $24 \mathrm{~h}$.

${ }^{\mathrm{b}}$ Calculated using univariate Poisson regression analysis.

c Univariate logistic regression analysis for dichotomous outcomes. 
Table 3 Degree of patent foramen ovale and risk of major decompression events (corrected for number of dives)

\begin{tabular}{llllll}
\hline & $\varnothing P F O$ & $\begin{array}{l}\text { Risk ratio with } \\
\text { PFO grade 1 }\end{array}$ & $\begin{array}{l}\text { Risk ratio with } \\
\text { PFO grade 2 }\end{array}$ & $\begin{array}{l}\text { Risk ratio with } \\
\text { PFO grade 3 }\end{array}$ & $\begin{array}{l}\text { Risk ratio per } \\
100 \text { dives }\end{array}$ \\
\hline $\begin{array}{l}\text { Number of divers } \\
\text { Major DCl }\end{array}$ & 167 & 13 & 27 & 23 & \\
$\begin{array}{l}\mathrm{DCl}>24 \mathrm{~h}^{\mathrm{a}} \\
\begin{array}{l}\mathrm{Stay} \text { in decompression } \\
\text { chamber }\end{array}\end{array}$ & - & $1.1(0.14-8.4)$ & $4.4(1.8-10.7)$ & $6.6(2.8-15.5)$ & $1.02(1.0-1.04)$ \\
\hline
\end{tabular}

Abbreviations: DCl: decompression illness, PFO: patent foramen ovale; ØРFO: no patent foramen ovale.

${ }^{a} \mathrm{DCl}>24 \mathrm{~h}=$ decompression illness lasting longer than $24 \mathrm{~h}$.

${ }^{\mathrm{b}}$ No diver in this group had a corresponding decompression event.

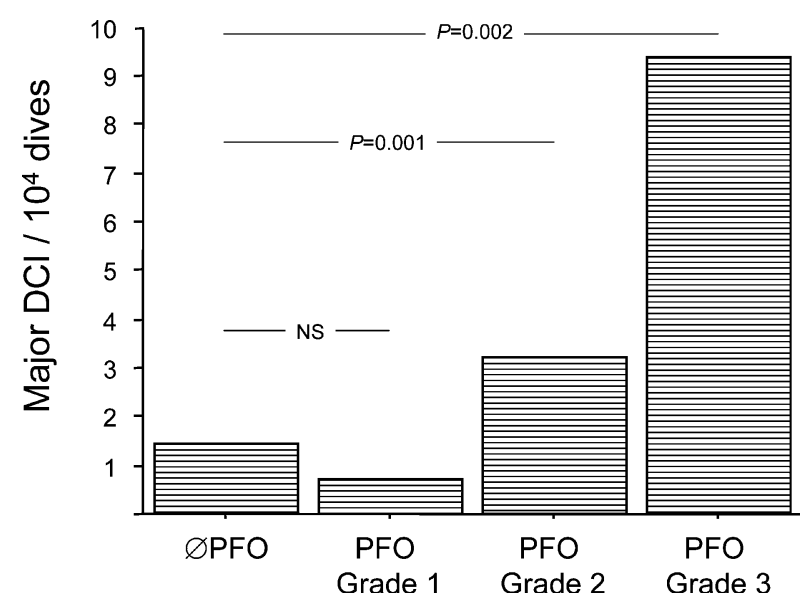

Fig. 3 Mean number of $\mathrm{DCl}$ events per $10^{4}$ dives (vertical axis) in relation to different sizes of patent foramen ovale (no PFO: $\varnothing \mathrm{PFO}=\mathrm{PFO}$ grade 0 ).

\section{Discussion}

The present study (the largest to date) in divers undergoing diagnostic testing for PFO revealed that the presence of TEE-detected PFO is related to an absolute risk of suffering approximately five major $\mathrm{DCl}$ events per $10^{4}$ dives, a risk which was found to be five times higher than that among divers without PFO. Using contrast TEE in this setting and addressing PFO size, a small PFO was found to behave like no PFO regarding the risk of $\mathrm{DCl}$ events, whereas large PFO's were discovered to bear the main risk, irrespective of further size distinction (grade 2 or 3 ).

\section{Decompression illness events: data from the literature}

Already in 1986, Wilmshurst and co-workers indicated that a cardiac right-to-left shunt may be relevant for paradoxical gas embolism in divers. ${ }^{4}$ In subsequent studies, the importance of PFO for decompression events has been further observed by investigating its presence among divers with and without a history of previous $\mathrm{DCl}$ events (up to $66 \%$ in the former versus $25-33 \%$ in the latter). ${ }^{5-7,9,10}$ These studies have major limitations however by being, in the majority too small and in one case exclusively including divers without $\mathrm{DCl},{ }^{10}$ by not taking into account the numbers of dives or having been pub- lished only in a preliminary form. ${ }^{9}$ Therefore, the actual risk of suffering an incident of $\mathrm{DCl}$ could have theoretically been determined only in the rather undersized study by Germopré et al., ${ }^{7}$ who, however, have refrained from doing so. In light of these explorations, our own published data, incorporating concrete risk assessment of $\mathrm{DCl}$ among 52 divers can also not be regarded as sufficiently substantial to settle the question whether, and exactly how often, the mentioned incidents occur depending on the presence or absence of PFO. ${ }^{11}$ The issue has also not been resolved by the recent meta-analysis ${ }^{8}$ which summarised some of the studies mentioned above ${ }^{6,9,10}$ and incorporated data on the number of dives from the general diving population, irrespective of PFO, in order to get an estimate of the risk of $\mathrm{DCl} .{ }^{13-16}$

At variance from the just mentioned investigations on the frequency of $\mathrm{DCl}$ without accounting for PFO, our study population consists of a mix between police, sport and commercial divers, who underwent a total of almost 175000 dives. Overall, the incidence of major $\mathrm{DCl}$ of 2.5 per $10^{4}$ dives in our investigation compares favourably with the respective average between military and sport divers of 1.9 per $10^{4}$ dives in previous studies (commercial divers accounted only for about a dozen in our study). ${ }^{13-15}$ The differences between incidences become larger if all three sub-groups of divers (sport, commercial and military divers) are considered, and they even reach one order of magnitude if all incidents are compared between commercial divers reported in the literature ${ }^{16}$ ( 35.3 per $10^{4}$ dive) and our entire population ( 2.5 per $10^{4}$ dives). These observed differences are likely to be artefacts (i.e., actual zero differences between rather small numbers), inaccuracies being incorporated in the data due to definition problems of decompression sickness, arterial gas embolism and $\mathrm{DCl}$, and due to self-reporting of the incidents by the divers. Regarding the definitions, it is quite difficult to distinguish decompression sickness from arterial gas embolism on the basis of the information provided by the diver about an event which took place at some point in the past. Therefore, we have decided to use the broader term $\mathrm{DCl}$ instead of decompression sickness and arterial gas embolism.

\section{Duration and treatment of $\mathrm{DCl}$ in relation to $\mathrm{PFO}$}

Two ways to partly compensate for the bias of self-reporting DCl event consist of obtaining "firm" rather than 
"soft" information, and of taking into account medical action taken in response to the incident into account. We hypothesised that the memory for severe neurological symptoms accounting for a major $\mathrm{DCl}$ and symptoms lasting longer than $24 \mathrm{~h}$ must be pre-eminent and less affected by gaps than other details of reporting such as e.g., the exact onset of symptoms (reportedly all within 30 min of surfacing) or the frequency of minor $\mathrm{DCl}$ signs. We therefore refrained from further analysing minor $\mathrm{DCl}$ symptoms and restricted our risk calculation on serious $\mathrm{DCl}$ incidents.

Along similar lines of reasoning, the fact that a serious $\mathrm{DCl}$ victim had to be treated in the decompression chamber obviously weighs much heavier than the information of the actual event itself, despite the much lower absolute number of decompression chamber stays. Information which has not been available so far is the relative risk of being treated by artificial recompression in a diver with PFO was 13 times higher than in one without PFO. One could argue that the decision of a physician to treat the diver's event by recompression could still be heavily influenced by the self-reporting and is, thus, not independent. This raises the question whether our study can provide other evidence supporting even a causal relation between PFO and DCI.

\section{Risk of $\mathrm{DCl}$ and size of PFO}

A dose-response relation between PFO and major DCI events would back their, at least partial, causal link. The "dose" or size of a PFO can actually be determined by using only TEE. Previous studies on the subject, with the exception of one, ${ }^{7}$ have employed the detection of echocontrast micro-bubbles in the systemic circulation from a transcranial or transthoracic site, both methods of which are much less sensitive than contrast TEE. ${ }^{17}$ Since the study by Hagen et al., in post-mortem hearts, the prevalence of PFO has been considered to be, on average, $27 \%$ across all age groups from the 1st to the 10th decade of life; in the 4th and 5th decade (the age groups of our population), it has been found to be $25 \%$ and $26 \%$, respectively. ${ }^{3}$ Using TEE, the prevalence in the present study was $27 \%$, reflecting the expected prevalence in the general population and ruling out selection bias. By using TEE for PFO sizing, a dose-response relationship between PFO and serious DCI was found (Fig. 3). The measurement of the size of a PFO using contrast bubbles is not quantitative but just a qualitative estimate of the amount of bubbles crossing the inter-atrial septum from right to left at the site of the PFO (Fig. 1). However, using stringent and systematically applied criteria for provoking a short rise in right atrial pressure (i.e., the Valsalva manoeuvre as described in the methods section) and for determining the time window of the bubbles' arrival in the left atrium (within four heart beats for the differentiation against transpulmonary shunts ${ }^{12}$ ) in the fully awake individual, the distinction between a small and large PFO is likely to be reliable. The only non-accounted for factor that could possibly be influencing the amount of crossing bubbles is a large Eustachian valve causing heavy streaming of bubble-free blood from the inferior vena cava with "washout" of bubble-rich blood arriving in the right atrium from the superior vena cava (Fig. 1(d)).

\section{Confounding variables}

Other factors, theoretically confounding the study results comprise differences in diving habits among the divers with and without PFO, such as a trend towards more frequent use of the Valsalva manoeuvre for pressure equilibration during ascent in the PFO group, the performance of more deep dives and the less frequent use of compressed air as breathing gas among divers with, rather than those without, PFO. Based on very scarce data in the literature on an increased frequency of PFO among, e.g., jazz saxophone versus bass players, ${ }^{18}$ it can be speculated that divers more frequently employing the Valsalva manoeuvre during ascent actually opened the PFO in the past. Considering the fact that divers not strictly adhering to decompression tables were excluded from this study, it would be similarly speculative to suppose that divers with PFO had more $\mathrm{DCl}$ events because they engaged more often in deep and thus more dangerous dives than divers without PFO. Conversely, the more frequent use of breathing gas containing higherthan-normal oxygen contents in divers unaware of their PFO indicates a kind of self-treatment of $\mathrm{DCl}$ and does not increase but rather prevent it.

\section{Clinical implications}

For all practical purposes, our study implies the following: the absolute risk of suffering a major $\mathrm{DCl}$ event is quite low even in divers with PFO and their five-fold higher risk than in those without PFO. However, the present study did not address the potential long-term neurologic hazards ${ }^{19}$ being inflicted by asymptomatic ischaemic brain lesions which have also been found to be related to PFO in divers, ${ }^{11}$ i.e., the effect of the cumulative cerebral ischaemic burden associated with PFO in divers is unknown. Accordingly, we advise a diver with a 2nd or 3rd degree PFO to refrain from diving. Theoretically, percutaneous PFO closure by a device could be efficacious in lowering the risk of $\mathrm{DCl}$ in divers, but this hypothesis has not been properly tested yet. The finding of our study that a small PFO behaves like no PFO regarding the frequency of serious $\mathrm{DCl}$, renders us to be less strict in our recommendation for refraining from diving. However, in a person with small PFO having suffered an incident of major $\mathrm{DCl}$, our recommendation is as follows: strict adherence to decompression tables, no deep dives (below $25-30 \mathrm{~m}$ ), no repetitive dives during a single day, reduced rate of ascent during the last $10 \mathrm{~m}$ below surface, no Valsalva manoeuvre during ascent, no strenuous physical effort shortly after leaving the water, and use of nitrox instead of compressed air. ${ }^{20}$

\section{References}

1. Weathersby PK, Homer LD, Flynn ET. Homogeneous nucleation of gas bubbles in vivo. J Appl Physiol 1982;53:940-6. 
2. Dutka AJ, Francis TJ. Pathophysiology of decompression sickness. In: Bove AA, editor. Bove and Davis' diving medicine. Philadelphia, PA: WB Saunders; 1997.

3. Hagen PT, Scholz DG, Edwards WD. Incidence and size of patent foramen ovale during the first 10 decades of life: an autopsy study of 965 normal hearts. Mayo Clin Proc 1984;59: 17-20.

4. Wilmshurst PT, Ellis BG, Jenkins BS. Paradoxical gas embolism in a scuba diver with an atrial septal defect. Brit Med J 1986;293: 1277.

5. Moon RE, Camporesi EM, Kisslo JA. Patent foramen ovale and decompression sickness in divers. Lancet 1989;1:513-4.

6. Wilmshurst PT, Byrne JC, Webb-Peploe MM. Relation between interatrial shunts and decompression sickness in divers. Lancet 1989;2:1302-5.

7. Germonpré $P$, Dendale $P$, Unger $P$ et al. Patent foramen ovale and decompression sickness in sports divers. $J$ Appl Physiol 1998;84:1622-6.

8. Bove AA. Risk of decompression sickness with patent foramen ovale. Undersea Hyperb Med 1998;25:175-8.

9. Moon RE, Kisslo JA, Massey EW et al. Patent foramen ovale (PFO) and decompression illness. Undersea Biomed Res 1991;13(Suppl.):15 (abstract).

10. Cross SJ, Evans SA, Thomson LF et al. Safety of subaqua diving with a patent foramen ovale. Brit Med J 1992;22:481-2.

11. Schwerzmann M, Seiler C, Lipp E et al. Relation between directly detected patent foramen ovale and ischemic brain lesions in sport divers. Ann Intern Med 2001;134:21-4.
12. Shub C, Tajik AJ, Seward JB et al. Detecting intra-pulmonary rightto-left shunt with contrast echocardiography. Observations in a patient with diffuse pulmonary arteriovenous fistulas. Mayo Clin Proc 1976;51:81-4.

13. Giliam BC. Evaluation of decompression sickness incidence in multiday repetitive diving for 77,680 sport dives. In: Lang MA, Vann RD, editors. Repetitive diving workshop. Costa Mesa, CA: American Academy of Underwater Sciences; 1990. p. 15-25.

14. Report on diving accidents and fatalities. Divers Alert Network; 1996.

15. Howsare CR, Jackson RL, Rocca AF et al. Navy decompression illness and fatalities. Undersea Hyperb Med 1997;24(Suppl.):77.

16. Imbert JP, Fructus $X$, Montbaron S. Short and repetitive decompressions in air diving procedures: the commercial diving experience. In: Lang MA, Vann RD, editors. Repetitive diving workshop. Costa Mesa, CA: American Academy of Underwater Sciences; 1990. p. $63-72$.

17. Di Tullio $M$, Sacco RL, Venketasubramanian $\mathrm{N}$ et al. Comparison of diagnostic techniques for the detection of patent foramen ovale in stroke patients. Stroke 1993;24:1020-4.

18. Kinra S, Okasha M. Unsafe sax: cohort study of the impact of too much sax on the mortality of famous jazz musicians. Brit Med $J$ 1999;319:1612-3.

19. Tetzlaff K, Friege L, Hutzelmann A et al. Magnetic resonance signal abnormalities and neuropsychological deficits in elderly compressedair divers. Eur Neurol 1999;42:194-9.

20. Schwerzmann M, Seiler C. Recreational scuba diving, patent foramen ovale and their associated risks. Swiss Med Wkly 2001;131:365-74. 